\title{
Improved Anterior Approach Offers Easy Access to Preperitoneal Space during Open Surgery of Groin Hernia Repair
}

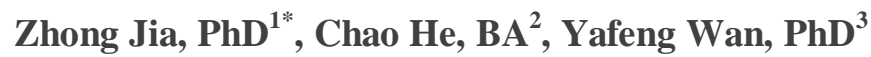 \\ ${ }^{1}$ Department of Hepatobiliary Surgery, Hangzhou Huansha Road 261,310006,10th Floor of No.1 building of Ha \\ ngzhou First People's Hospital,ZhejiangProvince, China \\ ${ }^{2,3}$ Department of Hepatobiliary Surgery, Hangzhou, First People's Hospital, Nanjing Medical University, \\ Affiliated Hangzhou Hospital \& Zhejiang Chinese Mediciene Affiliated 4th Clinical Teaching Hospital, \\ Hangzhou 310006, China \\ Co-first author: Chao He
}

*Coressponding Author: Zhong Jia, Department of Hepatobiliary Surgery,Hangzhou Huansha Road 261,310006,10th Floor of No.1 building of Hangzhou First People's Hospital,ZhejiangProvince, China. Email: jiazhong20058@ hotmail.com

\begin{abstract}
Background: There are many widely-used methods to perform groin hernia repair via anterior approach during open surgery. However, hernia-roof approach, aiming to let true hernia sac reduce to preperitoneal space naturally, lacks enough attention.

Objective: To explore the accessibility and value of hernia-roof approach during groin hernia repair.

Methods: 200 patients who underwent open groin hernia repair with anterior pathway to preperitonea approach between Jan. 2010 and Dec.2012 were retrospectively randomized selected as cases in the clinical trial, which were divided into two groups:A group (control group) adopted neck-shoulder technique $(n=100)$ and B group (study group) adopted hernia-roof technique(n=100).Their satisfactory rate, surgical-related complications, readmission rate within 1 month after discharge, recurrence rate within 1 year and reoperation within 3-year follow-up were collected and analyzed by software (SPSS 23.0 version, USA).All patients implanted the same material (SanShi Mesh, China).
\end{abstract}

Results: Two groups unveiled no statistic significance $(P>0.05)$.

Conclusion: Hernia-roof anterior pathway to entering peritoneal space is accessible and safe.The general value equals to neck-shoulder pathway but produces little damage to inner orifice.

Keywords: Groin hernia repair; Hernia-roof; Inner orifice; Preperitoneal approach

\section{INTRODUCTION}

The preperitoneal approach in open inguinal hernia repair surgery is widely applied in China. There are many anterior pathways to access the preperitoneal space such as laparoscopic techniques and neck-shoulder technique in open surgery. However, the integrity of groin area, including transversalis fascia, inner orifice, etc., seems not to be protected well particularly in open ways. How to gain an easy access to place mesh and reduce potential damage to normal tissue, will reduce discomfort risks after surgery such as bulging, chronic pain, etc., which however is given far little attention. In addition, it actually produces potential of intestinal injury when facing complex situations such as visceral adhesion or sliding hernia [1]. Now it is time to stress upon these loopholes and provide novel ways to resolve them. Therefore we would like to introduce hernia-roof technique to avoid these risks while the resistant degree of groin area strengthened.

\section{MAterial AND Methods}

\subsection{Surgical Procedures of Hernia-roof Technique}

The technique is performed under general or epidural or local anesthesia. A soft, light flat 
mesh in size of $10 \mathrm{~cm} \mathrm{X} 15 \mathrm{~cm}$ (San Shi Mesh, made in China) was used in all cases. If necessary, Foley catheter was placed as preoperation. The inguinal incision was of the length about $3 \sim 4 \mathrm{~cm}$. As usual, subcutaneous fat, external oblique muscle aponeurosis in turn was open, after that, the spermatic cord and bulging hernia were separated till whole hernia was seen. The key steps includes 3 parts:

To expose inguinal hernia and open the outside of hernia layer by layer just at the position of hernia-roof till the true sac was seen. The inner orifice had been fully exposed after the outside part of true sac was totally stripped to the bottom of sac neck.

The preperitoneal space can be easily detected without further separation if you grip the true sac with oval clip and push through inner orifice. In general, the central point of mesh was fixed on the true sac-roof by suture.

Using an oval clamp or forceps with blunt top end to grip the edge of wrapped mesh, and then push mesh along with the true sac itself both into the targeted preperitoneal field. If the size of inner orifice was bigger enough to pass through a finger, mesh can be flatted with finger. Reversely, defined forcep was needed to help mesh pass through the inner orifice. Also flatten mesh had better be done carefully with a little forcep .Likewise another side of mesh was done.

Inner orifice was closed and fixed upon the mesh. In the end, mesh, true sac, and inner orifice were forged a unit. The extra leash along with tedious residual of the outside cover was removed away. If any, upper mesh had better been inserted between transversalis fascia and combined tender. If necessary, it's good to place a drainage tube, aiming to prevent the potential of seroma.

\subsection{Trial Design}

From year 2010 to year 2012,200 patients who underwent open groin hernia repair via open anterior approach were retrospectively collected ,among of whom, 100 cases adopted hernia-roof technique, another 100 cases adopted neckshoulder technique. The former was divided as study group B while the later control group A. In this trial ,postoperative complication, readmission within 1 month, recurrence within 1 year, chronic pain or discomfort of bulging, reoperation within 3 years were chosen as comparative data.

\section{RESULTS}

In group A, surgical site infection was found in 3 cases, recurrence of hernia was found in 2 cases, discomfort of bulging was found in 5 cases ; In group B, surgical site infection was found in 2 cases, recurrence of hernia was found in only 1 case, discomfort of bulging was found in only one case, one of case died of heart stroke during 2-year follow-up. No readmission, reoperation, chronic pain was found within 3year follow-up. Statistic analysis (SPSS 23.0 version, $\mathrm{X} 2$ test) showed no significant difference between group A and B.

\section{DISCUSSION}

Inguinal hernia is one of the most common diseases in China. The incidence is approximately $0.3 \% \sim 0.5 \%$, and $1.16 \%$ in elderage males ( $>60$ years). It is a big challenge to repair the weak groin area of an elderly patient with huge groin hernia. In addition, majority of the elder have chronic cough, coronary heart disease, serious constipation, and other comorbidities. As a result, the groin area is more likely to bulge or face recurrence of hernia. Generally, the recurrence rate of groin hernia after surgery in China is approximately up to1 2\%. In addition, the other operative complications such as nerve injury-originated pain and discomfort of bulging are still exist when the patients are followed up in the doctors' office. In the past two decades, the preperitoneal approach to groin hernia repair using a flat mesh or plug has become worldwide used [2]. As we all well know, it is crucial key to locate the defect in the transversalis fascia and then to remedy it. In 2010, Mainik etc. [3] reported the preperitoneal loop found unexpectedly in the total extraperitoneal herniorrhaphy approach. Actually, the preperitoneal loop is just the inner ring (inner orifice). The key to tension-free inguinal herniorrhaphy is not only to make up for the defect in the transversalis fascia but also to intensify its resistant degree with artificial mesh. In addition, keeping the integrity of inner orifice is another key to success. The edge of inner orifice is often thickened due to repeated protrusion of hernia, which produces the feature of flexibility. The inner orifice is just like a buttonhole of a cloth, if the hole is not protected properly, the cloth is more likely to be damaged. The most value of hernia-roof technique is to protect the inner orifice, which gets positive effects similar to protection of the buttonhole of 
a cloth. It is accessible and reliable according to our study.

The transversalis fascia actually consists of two layers including the deep one and superficial one. The superficial layer is equal to the outer side cover of true sac, and the deep layer is equal to the level of inner orifice. This basic anatomic knowledge will help surgeons better understand hernia-roof pathway. In general, a flat, soft, and light-weight lower mesh or plug is easy to be pushed through the inner orifice into the preperitoneal space if the inner orifice is big enough, but it is difficult to do so as the inner orifice is small(size less than $1.5 \mathrm{~cm}$ ). Based on previous neck-shoulder technique, the inner orifice is often damaged so as to get an access to preperitoneal space. Obviously, the demand of protection of the inner orifice is in need, if Hernia-roof technique provides an alternative choice to settle these problems. During the surgery, the lower mesh is fixed on the herniaroof and the inner orifice simultaneously. Patients could ambulate immediately and all patients were satisfied and recovered uneventfully. The comparative study further confirms the value of hernia-roof technique [47]. As a note, the hernia sac should be pinched by fingers prior to incise the outside cover of hernia sac so as to prevent the real sac sliding into the preperitoneal space or injured accidently. The improved techniques are easy to understand and to learn for mostly surgeons. However, if the outside cover of hernia sac is not so easy to be taken off , the other approaches should be considered without any hesitation. It is necessary to place negative pressure device to drain the potential of fluid accumulation during emergent surgery.

\section{Conclusions}

Hernia-roof technique provides a stronger groin area via pining closure of hernia orifice, true sac, mesh together. The value at least equals to neck-shoulder technique.

\section{ACKNOWLEDGEMENT}

The study was sponsored by Hangzhou Science and Technology Program, Zhejiang Province, China (No.20150733004)

\section{REFERENCES}

[1] Cavazzola LT, Rosen MJ. Laparoscopic versus open inguinal hernia repair.SurgClin North Am 2013;93:1269-79.

doi: 10.1016/j.suc.2013.06.013.

[2] Basile F, Biondi A, DonatiM.Surgical approach to abdominal wall defects: history and new trends. Int J Surg 2013;1:S20-3. doi: 10.1016/S1743-9191(13)60008-4

[3] Mainik F, Quast G, Flade-Kuthe R, Kuthe A, SchroedlF.Thepreperitoneal loop in inguinal hernia repair following the articletotallyextraperitonealtechnique.Hernia 2010;14:361-7. doi: 10.1007/s10029-010-06445.

[4] Jia Z,WangP,ZhuYH,WuK.Approach from hernia-roof:a new technique of preperitoneal tension-free inguinal hernia repair.Chin J Hernia Abdominal WallSurg02015;9:57-58.doi: 10.3877/cma.j.issn.1674-392X.2015.04.017

[5] Wu K,JiaZ,Zhu YH.3-D Herniorrhaphy may be Accessible via Hernia Sac Top Pathway. Surgery Curr Res 2016;6:272.doi: 10.4172/21611076.1000272

[6] $\mathrm{Wu} \mathrm{K,JiaZ,Jin} \mathrm{HC}$ et al.Anatomical basis and clinical application of preperitoneal tension-free hernia repair via HST approach.Chin J Hernia Abdominal Wall Surg 2016;10:369-71. doi: 10.3877/cma.j.issn.1674-392X.2016.05.014

[7] Wang J,ZhuYH,Jia Z*.A case of hepatic cirrhosis combined with ascites and incarcerated hernia by preperitoneal approach repair through Hernia Sac Top Pathway.IntSurg J 2016;3(1):372-376.doi: $\quad 10.18203 / 2349$ 2902.isj20160262.

Citation: Zhong Jia, Chao He \& Yafeng Wan. Improved Anterior Approach Offers Easy Access to Preperitoneal Space during Open Surgery of Groin Hernia Repair. ARC Journal of Surgery.2018; 4(1):6-8. doi: dx.doi.org/ 10.20431/2455-572X. 0401002.

Copyright: (c) 2018 Authors. This is an open-access article distributed under the terms of the Creative Commons Attribution License, which permits unrestricted use, distribution, and reproduction in any medium, provided the original author and source are credited. 